\title{
How far in-silico computing meets real experiments. A study on the structure and dynamics of spin labeled vinculin tail protein by molecular dynamics simulations and EPR spectroscopy
}

\author{
MNV Prasad Gajula ${ }^{*}$, KP Vogel$^{2}$, Anil Rai ${ }^{1}$, Franziska Dietrich ${ }^{3}$, HJ Steinhoff $^{2}$
}

From ISCB-Asia 2012

Shenzhen, China. 17-19 December 2012

\begin{abstract}
Background: Investigation of conformational changes in a protein is a prerequisite to understand its biological function. To explore these conformational changes in proteins we developed a strategy with the combination of molecular dynamics (MD) simulations and electron paramagnetic resonance (EPR) spectroscopy. The major goal of this work is to investigate how far computer simulations can meet the experiments.

Methods: Vinculin tail protein is chosen as a model system as conformational changes within the vinculin protein are believed to be important for its biological function at the sites of cell adhesion. MD simulations were performed on vinculin tail protein both in water and in vacuo environments. EPR experimental data is compared with those of the simulated data for corresponding spin label positions.

Results: The calculated EPR spectra from MD simulations trajectories of selected spin labelled positions are comparable to experimental EPR spectra. The results show that the information contained in the spin label mobility provides a powerful means of mapping protein folds and their conformational changes.

Conclusions: The results suggest the localization of dynamic and flexible regions of the vinculin tail protein. This study shows MD simulations can be used as a complementary tool to interpret experimental EPR data.
\end{abstract}

\section{Background}

Rapid advances in computer technology have led to the development of successful molecular simulations of protein structural dynamics that are intrinsic to understand biological processes. These simulations have resulted in the development of novel models and methods that increasingly agree with experimental observations, and suggest new experiments providing insights into biological mechanisms. Used in combination with the information

\footnotetext{
* Correspondence: gajula@iasri.res.in

${ }^{1}$ CABin division, DST Ramanujan Fellow, Indian Agricultural Statistics

Research Institute, PUSA campus, New Delhi-110012, India

Full list of author information is available at the end of the article
}

gained by sophisticated experimental techniques, molecular simulations can help us, to understand biological complexity at the atomic and molecular levels. Here, we emphasize such an approach that illustrates the potential of molecular dynamics simulations in analyzing experimental results determined by EPR spectroscopy on vinculin as an example.

Vinculin is a highly conserved intracellular protein that plays a critical role in cellular adhesion, migration, maintenance and regulation of cell shape [1-3]. Vinculin exists in two distinct conformations depending on an intra-molecular interaction between its head $(\mathrm{Vh})$ and tail (Vt) domains (cf. Figure 1) [4-6]. Conformational changes

\section{Ciomed Central}




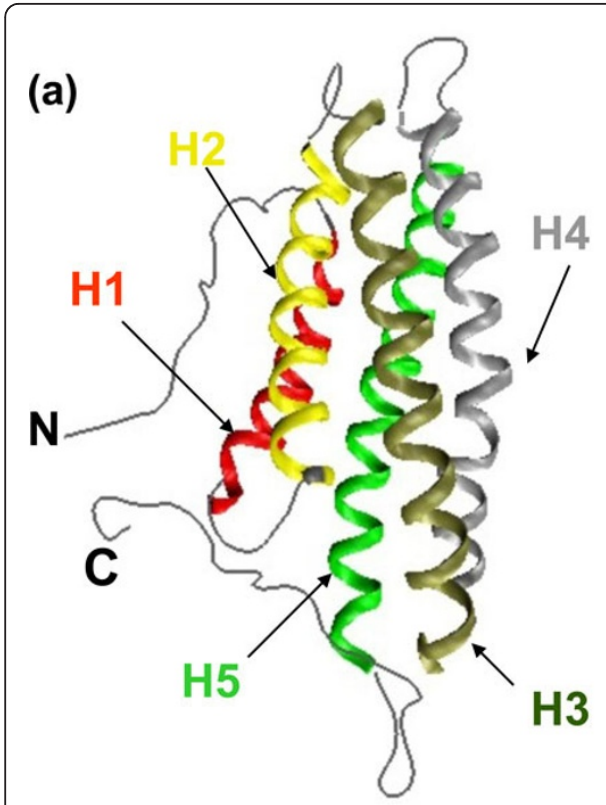

$\mathrm{H} 1$ = residues $895-913$

$\mathrm{H} 2=$ residues $919-939$

$\mathrm{H} 3=$ residues $945-973$

H4 = residues $979-1006$

H5 = residues $1013-1047$

Hydrophobic hairpin= 1062-1066 $\left(b^{*}\right)$

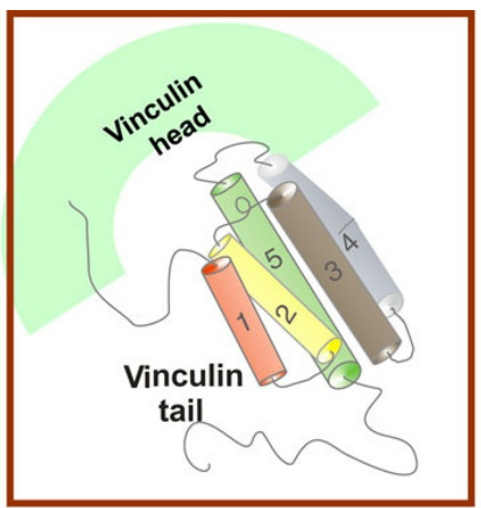

\section{Inactive state}

$$
\downarrow
$$

Active state

\section{Cell membrane}

Figure 1 Schematic representation of structure and function of vinculine tail. (a) Vinculin tail domain with its 5 helix pack; Each helix is shown in different color for easy identification. $\mathrm{N}$ and C-terminal domains are shown as threads. The residue numbers of each helix are given. (b) Schematic representation of the functional domains of vinculin and some of its ligands. Vinculin is supposed to cycle between active and inactive conformations. Insertion of the hydrophobic hairpin(C-terminal) into the membrane is thought to be the primary step involved in the $\mathrm{Vt}$ detachment from its head. (b)(top) Inactive state of the vinculin is shown in box. In this state, vinculin is held in a "closed", auto-inhibited conformation by intra-molecular interactions between the head and tail domains. (b)(bottom) A model of vinculin activation. Conformational changes in the C-terminal tail domain are thought to play a key role in this action, but there are no significant studies so far that provide the active state conformation in living cells. ${ }^{*}$ Inspired from [5,6]. For more extensive reviews also refer to [3,28].

within the vinculin protein are believed to be important for its biological function [7-9]. Despite intensive biophysical and biochemical studies, the dynamics of vinculin activation are still unclear [10-14]. Conformational changes in the $\mathrm{C}$-terminal tail domain are thought to play a key role in this action. Here, we employ molecular dynamics simulations to study the site specific behaviour of the vinculin tail in its inactive conformation. We focus on the dynamics of spin labels that are bound to specific protein regions and which could provide insight into possible conformational changes during protein function.

Site directed spin labeling EPR spectroscopy has evolved as a powerful technique to investigate protein structure and conformational changes under physiological or near-physiological conditions [15-18]. The shape of continuous wave (cw) EPR spectra recorded at room temperature is sensitive to the re-orientational motion of the bound spin label side chain providing information on the motional restriction of the nitroxide due to sterical interaction with the secondary and tertiary structure [18-20]. In addition, solvent accessibility of the spin label side chain and polarity of the nitroxide microenvironment characterize the protein topology. Dipolar coupling between two spin labels incorporated into a protein report on intra-molecular distances [21,23]. Details of this method are summarized in recent reviews by Bordgnon et al, and Klare et al. Here we report on 
MD simulations of spin labelled vinculin tail in order to analyze experimental spin label EPR spectra.

\section{Methods}

The initial coordinates for the vinculin tail MD simulations were obtained from the Protein Data Bank (PDB code: 1ST6). All MD simulations were performed with the GROMACS simulation suite for in water and in vacuo simulations. The force field ffG43a1 was used for the simulations in water and the force field ffG43b1 from GROMOS, which is integrated into GROMACS, was used for the simulations in vacuo. Several MD simulations were performed with different spin labeled sites for $30 \mathrm{~ns}$ at $300 \mathrm{~K}$ in water, whereas the simulations in vacuo were performed for $10 \mathrm{~ns}$ at $600 \mathrm{~K}$. In order to assure that the spin label covers the maximum accessible conformational space within a relative short MD run of $10 \mathrm{~ns}$, a high temperature of $600 \mathrm{~K}$ was applied with position restraints on all backbone atoms. Periodic boundary conditions were applied as mentioned elsewhere. For a detailed description of the MD simulations methodology and applications refer $[25,29,30]$. The vinculin tail protein holds two native cysteines at positions 950 and 972 on helix 3. Cysteines were genetically introduced at positions 901, 909 on helix 1 (H1); 922,927,934 on helix 2(H2); 957 on helix 3(H3); 984 on helix 4(H4); 1024, 1033 on helix 5(H5); and 1062 in the C-terminus. EPR experiments on these mutants were described in [20]. Refer to [24-26] for calculating EPR spectra from MD simulations data.

\section{Results}

\section{Spin label dynamics in water and in vacuo}

MD simulations were performed in water and in vacuo environments as described in the methods section on a series of selected spin labeled sites specifically bound to the vinculin tail protein. In order to characterize the dynamics of the spin-labeled side chains, the reorientational dynamics are represented by the mean fluctuation of the nitroxide ring orientation as shown in Figure 2. Here the angle $\beta$ is the angular deviation from the mode of the orientational distribution of the $\mathrm{z}$ - axis of the nitroxide spin label. (see [24-26] for more information about the angle $\beta$ ). As an example, the distributions of $\beta$ for only two spin labeled residues, positions 901 and 909 are shown in Figure 3, the widths of the distributions for all samples are depicted in Figure 4. The $\beta$ distribution widths determined from MD simulations in vacuo and in water show similar behaviour. In both cases the calculations reflect the dependency of the spin label mobility on the specific location.

\section{Mobility parameter}

For a quantitative comparison of experimental and simulated data we calculated mobility parameters
$[26,27]$. The mean square fluctuation amplitude of the nitroxide $(\langle\Delta \beta\rangle)^{2}$ is determined from the simulations. The inverse line width of the central resonance $\Delta \mathrm{H}_{0}^{-1}$ of EPR spectra is a convenient experimental measure of the nitroxide mobility that can be compared to the MD simulations results. The higher these mobility parameters, the higher the flexibility of the spin label side chain. The nitroxide mobility from the series of vinculin tail variants discussed above is projected in Figure 5 as function of residue number. The experimental values were taken from [20].

\section{Comparison of simulated and experimental data}

In addition to the reorientation angle analysis, EPR spectra were calculated from MD simulations data according to the method described in [24-27]. The simulated spectra from MD data were compared with those of experimental EPR spectra of spin labels attached to various positions of the vinculin tail that are presented in Figure 6. Most of the spectra show two distinct components that reflect the different dynamic modes of the spin label side chain depending on the interactions with nearby residues and helical contacts. In Figure 6, immobile and mobile componants are indicated by the red and gray bar respectively. In addition, a high mobility of the spin label may indicate a flexible region of the protein. The simulated spectra of spin labels at positions 901 to 922 show similar behaviour both in water and in vacuo simulations. The $\beta$ distributions (Figures 3 and 4) support this data. An experimental spectrum is available for position 922 that reveals a higher mobility compared to the simulated spectra. A different mobility pattern is seen in water and in vacuo simulations for the positions 927 and 934 . In vacuo simulations show a decrease in the mobility for both positions reflecting more backbone or tertiary interactions. Unfortunately, experimental spectra are not available for these two positions. 950R1 shows a similar behaviour both in water and in vacuo simulations. Again the experimental data reveal higher mobility. In contrast, the simulated spectra of the spin label at 957 show higher mobility than revealed in the experimental spectrum. Water simulations of the spin labels attached to 984 in helix 2 show similar behavior to the experimental spectrum, whereas the spectra determined from MD simulations performed in vacuo differ with that data. The spin label at position 1024 shows an increased mobility in both simulated and experimental spectra. A small immobile peak in the experimental spectrum suggests a significant interaction for this position with the surrounding residues. The simulated spectrum for 1033R1 in vacuo is well in agreement with that of the experimental spectrum with a decrease in mobility. Again the spin label at position 1062 shows similar behavior in both simulated and 


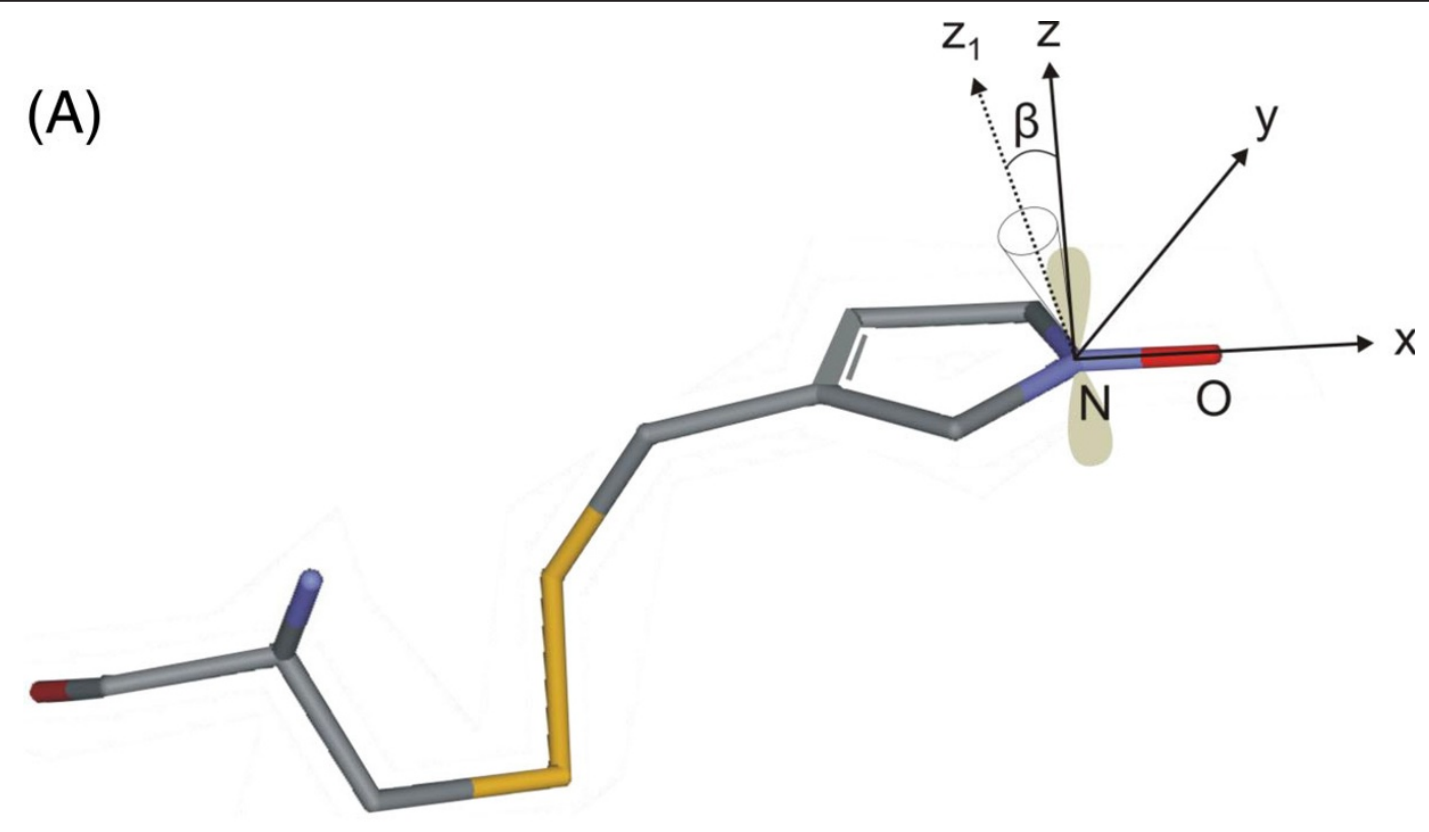

(B)
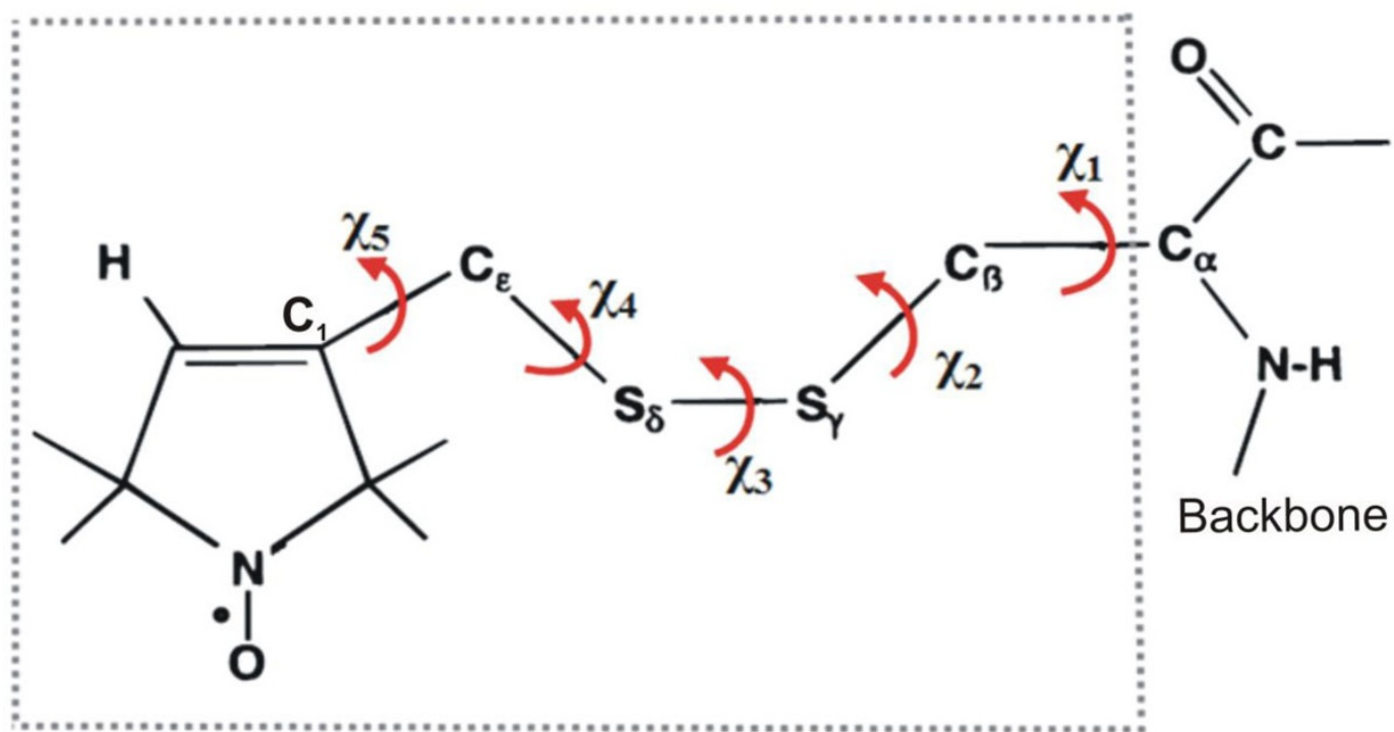

Figure 2 Spin label side chain. (a) Cysteine bound spin label MTSSL. The molecular coordinate system $(x, y, z)$ of a nitroxide with $x$ along the $\mathrm{N}-\mathrm{O}$ bond and $\mathrm{z}$ perpendicular to the plane of the nitroxide for z-axis anisotropic motion is depicted. The orientation of the tether with respect to the nitroxide axis system is determined by the angles $\alpha, \beta$ and $\gamma$ ( $\alpha$ and $\gamma$ not shown). $\beta$ is the angular deviation from the mode of the orientational distribution of the z-axis of the nitroxide. The nitrogen $p$ orbital along the z-axis is shown in shaded color. For simplicity, the methyl substituents of the nitroxide ring are not shown. (b) All flexible bonds within the spin label side chain (shown in dotted box). The interaction between the $S \gamma$ and $C \alpha$ proton limits the rotmers $C \alpha-C B, C B-S \gamma$, S $\gamma-S \delta$. 


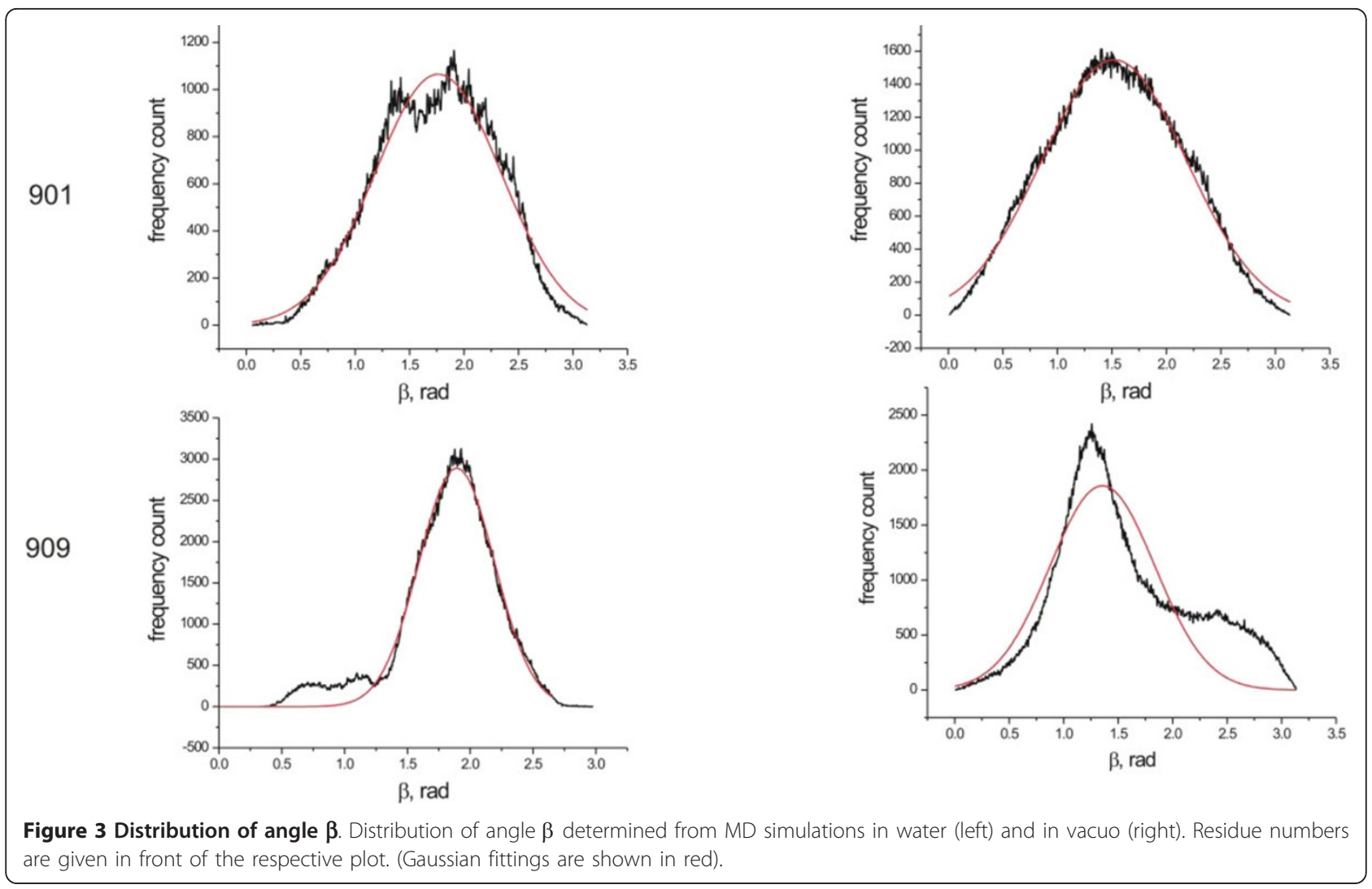

experimental spectra, with the experimental spectrum revealing less motional restrictions. When compared to the experimental data, the dynamics of the spin labels at positions 957, 1024 and 1033 show higher mobility in simulations, whereas the tendency is true for 922, 950, and 1062.

\section{Inter helical distances}

Inter helical distance measurements within the vinculin tail are good means of representing the tertiary structure. In addition, they give a highly localized view of the conformational dynamics of the polypeptide chains. The results presented in Figure 7 show the distances between selected spin labeled sites within the vinculin tail domain as simulated by in vacuo MD simulation. Sites were chosen according to available experimental data [20]. Experimental and simulated inter residue and inter nitroxide distances are listed in Table 1.

\section{Discussion}

The RMSF plot in Figure 8 shows that the trajectories are well equilibrated during the simulations. The helix, loops and terminal domains are clearly distinguished in the plot. It is interesting that the loops between $\mathrm{H} 1-\mathrm{H} 2$ and H3-H4 are not highly fluctuating compared to the other loops. The structural inspection suggests that these two loops are small in length and that the loop between $\mathrm{H} 1$ and $\mathrm{H} 2$ is masked by the long $\mathrm{C}$ - terminal end domain. The lower flexibility of the loop between $\mathrm{H} 3$ and $\mathrm{H} 4$ is associated with the central kink in helix $\mathrm{H} 4$ that brings one end of the $\mathrm{H} 4$ into strong tertiary and backbone contacts with helix 3 residues (see Figure 1).

The reorientational angle distribution for the spin label at position 901 (on H1 helix) in water and in vасио simulations is presented in Figure 3. Similar broad distributions are found for this position both in water and in vacuo simulations. This spin label is located near the flexible loop region and also the head group of the side chain is exposed to the surface of the protein. The spin label at position 909(H1) shows a narrow distribution of $\beta$ in water simulations as well as in vacuo simulations. This indicates that the spin label mobility at this position is restricted. Inspection of the structure indicates that this spin label is buried between two helices. The reorientational dynamics of the spin label at position $922(\mathrm{H} 2)$ is characterized by a broad distribution both in water and in vacuo simulations. This indicates that the spin label at this position is mobile. Though part of the spin label linker is buried, the nitroxide shows a significant mobility because the more flexible dihedrals $\chi 4$ and $\chi 5$ are not restricted (Figure 2). The spin label at position $927(\mathrm{H} 2)$ shows a moderate 


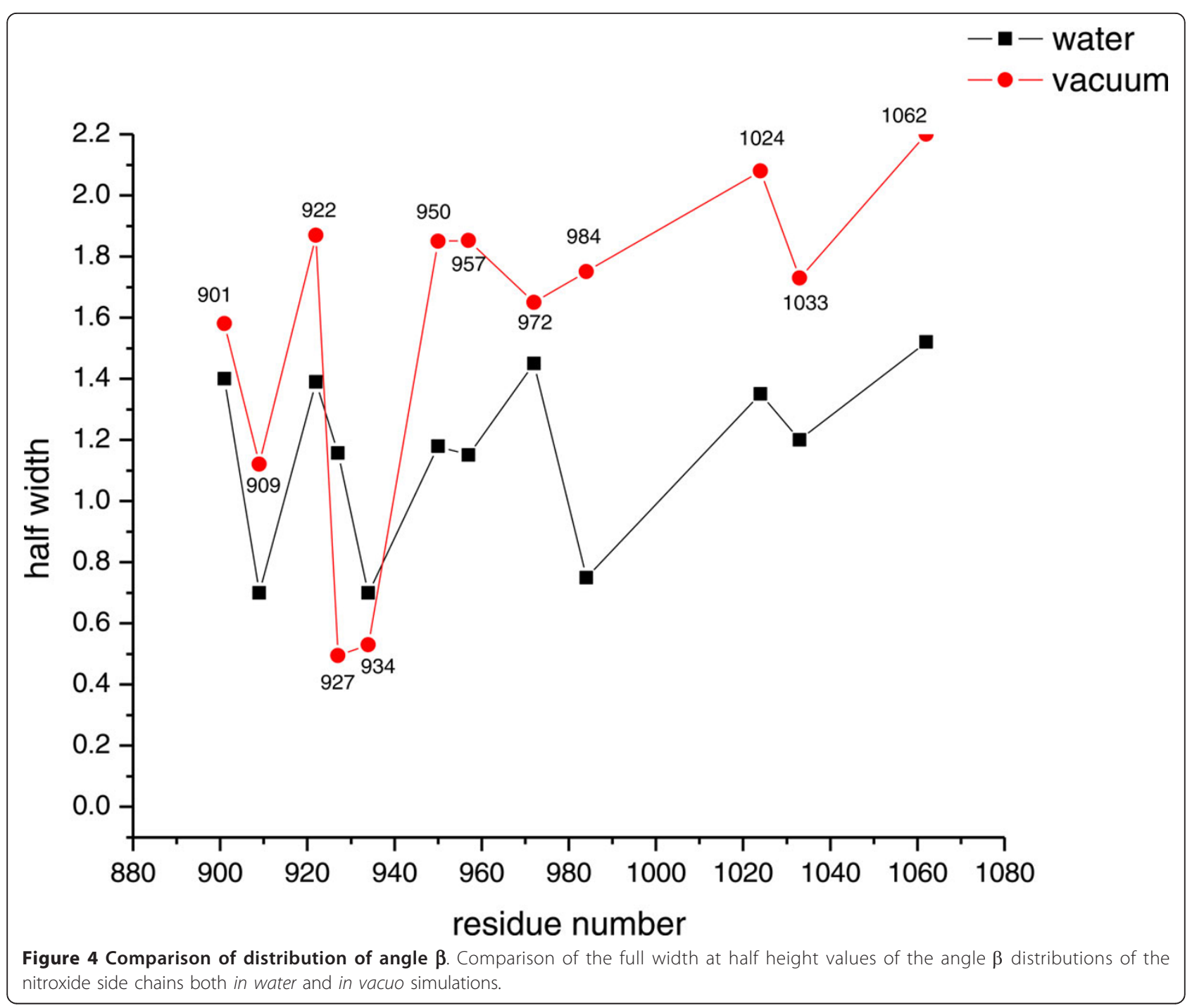

distribution of $\beta$ in water simulations when compared to the position 922(H2). However the distribution of $\beta$ in vacuo simulations indicates that the spin label at this position is highly restricted due to contacts with neighbouring helix atoms. The spin label at position 934(H2) shows restricted mobility, both in water and in vacuo simulations with narrow $\beta$ distributions. This spin label side chain is surrounded by bulky residues. Considering the overall mobility pattern, the $\mathrm{H} 2$ helix shows restricted mobility with low $\beta$ distributions.

Helix H3 carries three spin labels located at positions 950, 957 and 972. The distribution plots indicate that the mobility is moderate in water simulations, whereas in vacuo simulations the mobility is significantly increased. A very interesting distribution pattern is found in vacuo simulations of 972R1 that indicates two rotameric states of the spin label that are equally distributed (data not shown). This spin label is located near the loop region and oriented between two helices. However a functional study has shown a perturbed conformation of protein due to the bound spin label at position 972. Helix H4 has only one spin label at position 984 showing very narrow distribution of $\beta$ in water simulations and a larger distribution in vacuo simulations. This spin label location is near to the kink region of the helix. The narrow distribution of $\beta$ in water simulations reveals that this spin label has strong interactions during the simulations, whereas in the vacuo simulations the spin label is mobile. This indicates helical movement in the in water simulations that brings the spin label into strong tertiary contacts. H5 helix holds 1024R1 and 1033R1. Both MD simulations in water and in vacuo indicate that 1024R1 is very mobile when compared to 1033R1. 1024R1 is a surface exposed site. Though the spin label at 1033 is partially surface exposed, its mobility is restricted by the presence of an arginine residue located at 987 in the adjacent helix. 


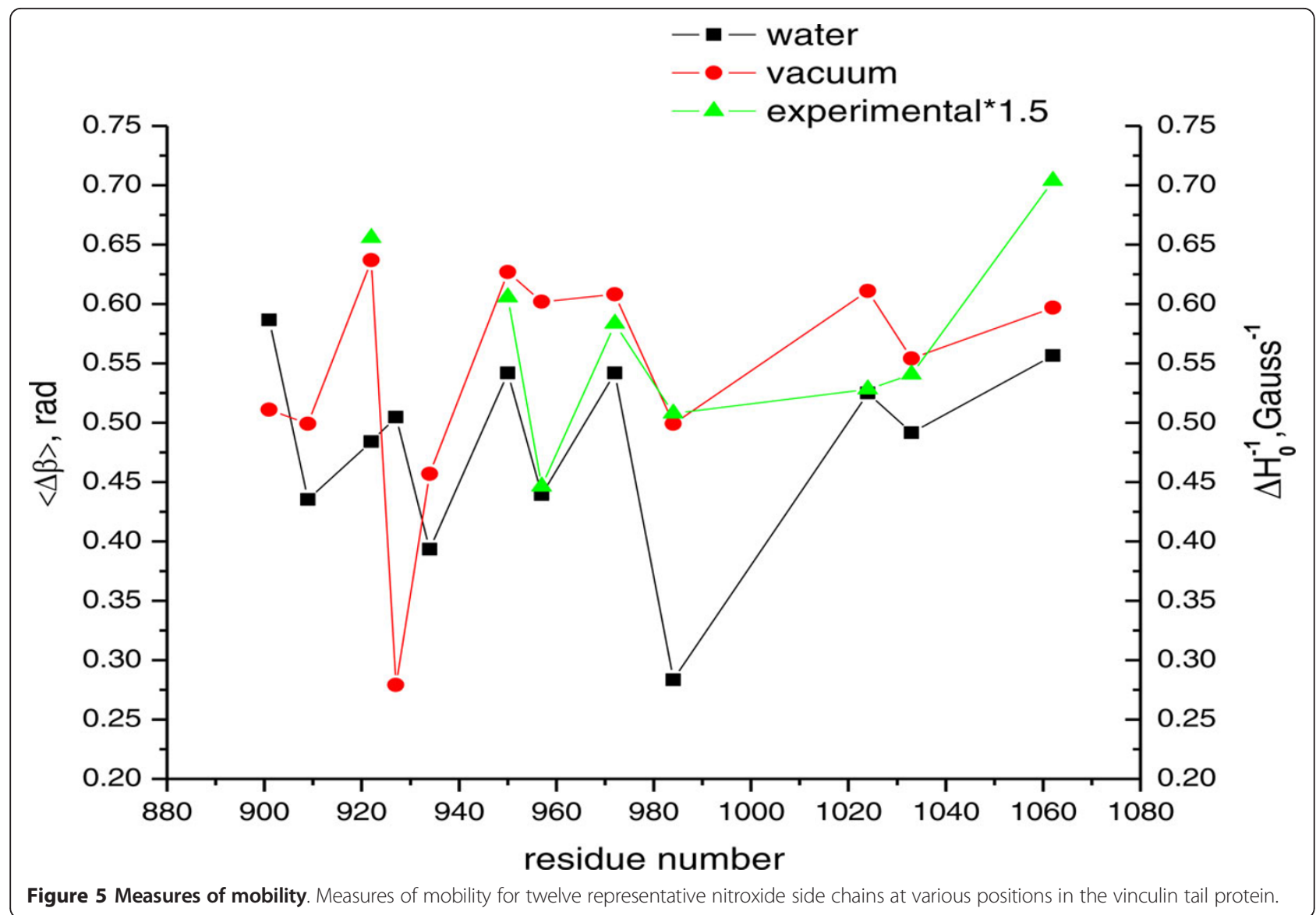

1062R1 is located on a $\mathrm{C}$-terminus end that naturally shows a broad distribution of $\beta$ in both in water and in vacuo simulations.

As can be seen from Figures 5 and 9 the overall mobility of the spin labels calculated from in vacuo simulations is larger than that determined from in water simulations. This is most probably due to the comparably large temperature of $600 \mathrm{~K}$ of the vacuo simulations which allows the spin label to overcome sterical barriers which may restrict the motion at $300 \mathrm{~K}$ in water. One exception is position 927 where the vacuo simulations reveal a highly restricted dynamics. This might be due to the fact that the backbone motion is restricted in vacuo simulations by positions restraints of $\mathrm{C} \alpha$ and this spin label is in strong contact to a neighboring helix which cannot move. Furthermore, with this exception, the overall pattern characterized by the relative changes of the mobility is similar in vacuo and in water simulations. This pattern is also reflected in the experimental mobility parameter for the sequence 950-984 and for 1033/1062. The color coding in Figure 9 shows that the spin labels in water environments exhibit a variety of mobility behaviour depending upon the label position along the protein chains.
From Figures 5 and 6, it is clear that high mobility is associated with residues 901, 922, 950, 972, 1024 and 1062. Among these spin labels, the one at position 1062 is in the $\mathrm{C}$-terminal region. 901R1 that belongs to $\mathrm{H} 1$ helix is immediately next to the loop region. Therefore it shows a high degree of freedom in water simulations.

The structural dynamics within a subunit of the vinculin tail protein depicted by the relative motion between spin labels 901 and 957 in helices $\mathrm{H} 1$ and $\mathrm{H} 3$ respectively is shown in Figure 7. The distance distribution shows the mean distance of $27.2 \AA$ with an overall width of about $2 \AA$. The distance values between spin labels at positions 922(H2) and 957(H3) reveal a smaller distribution. A study by Palmer et al [28] suggests that conformational change in the vinculin C-terminal may depend on a critical histidine residue at position 906 in H1. The conformational change triggered by the presence of this histidine may push/pull $\mathrm{H} 1$ with respect to H3. The distribution of distance between 901 and 1033 is similar to the distribution between 901 and 957. It is interesting that the distance distribution between 984R1 (H4) and 1033R1 (H5) shows two distinct maxima at $7 \AA$ and $9 \AA$ respectively. It indicates that one of the spin labels fluctuates around two conformations out of which the most 

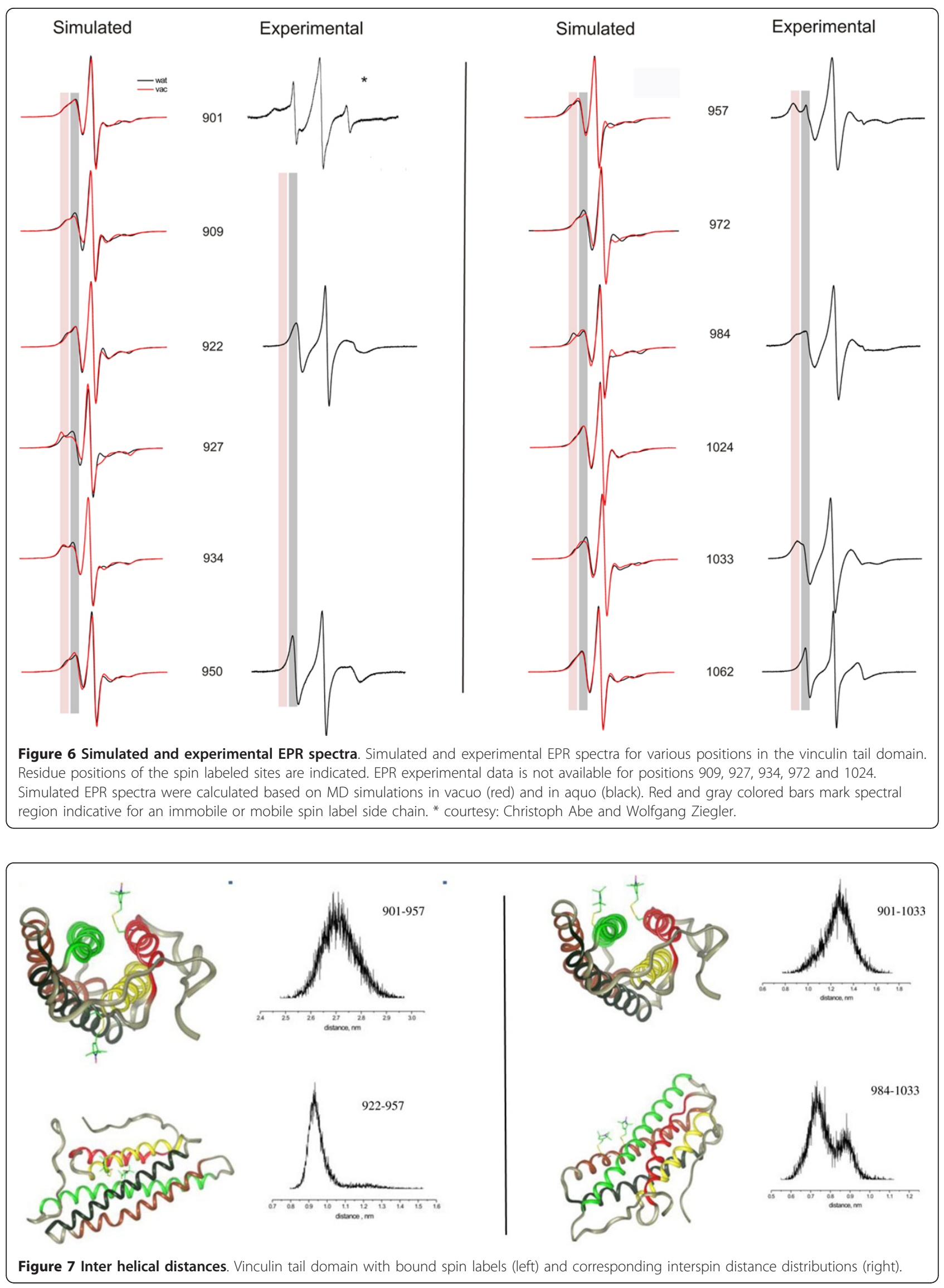
Table 1 Distance comparisons

\begin{tabular}{|c|c|c|c|}
\hline Residues & $\begin{array}{c}\text { X-ray } \\
(C \alpha-C \alpha), \AA\end{array}$ & $\begin{array}{c}M D \\
(N X-N X), \AA\end{array}$ & $\begin{array}{c}E P R \\
(N X-N X), \AA\end{array}$ \\
\hline $901-957$ & 16.4 & 27.2 & $26.5 \pm 1$ \\
\hline $922-957$ & 5.53 & 9.5 & $8 \pm 1$ \\
\hline 901-1033 & 11.37 & 12.7 & $8 \pm 1$ \\
\hline 984-1033 & 6.58 & $\begin{array}{c}7.3(\sim 65 \%) \\
9(\sim 35 \%)\end{array}$ & $7 \pm 1$ \\
\hline
\end{tabular}

Comparison of distances from X-ray structure, MD simulations and EPR experiments [20]

dominant conformation is at $7 \AA$. This is in agreement with the observation that the angle $\beta$ of 1033 shows two distinct maxima. The existence of multiple conformations in dynamic equilibrium also raises questions regarding the internal mobility of the individual side chains as well as of larger structural domains. However, the MD simulations data show that both these spin labeled positions show significant motional restriction. The simulated inter-nitroxide mean distances are in-line with the results of EPR experiments [20] (cf. Table 1).

The MD simulations data together with EPR results indicate, while the majority of the spin labeled sites in the vinculin tail show considerable dynamics, perhaps the most significant ones are those belonging to helices $\mathrm{H} 1, \mathrm{H} 3, \mathrm{H} 5$ and naturally the $\mathrm{C}$-terminal end residue 1062. (refer to [31-33]). This dynamics might be of importance for the understanding of the unfolding process of the vinculin tail bundle and its interaction with the membrane.

\section{Conclusions}

MD simulations were performed on the spin labeled vinculin tail domain both in vacuo and in water environment. The behavior of the spin label at various positions of the vinculin tail domain was analyzed by means

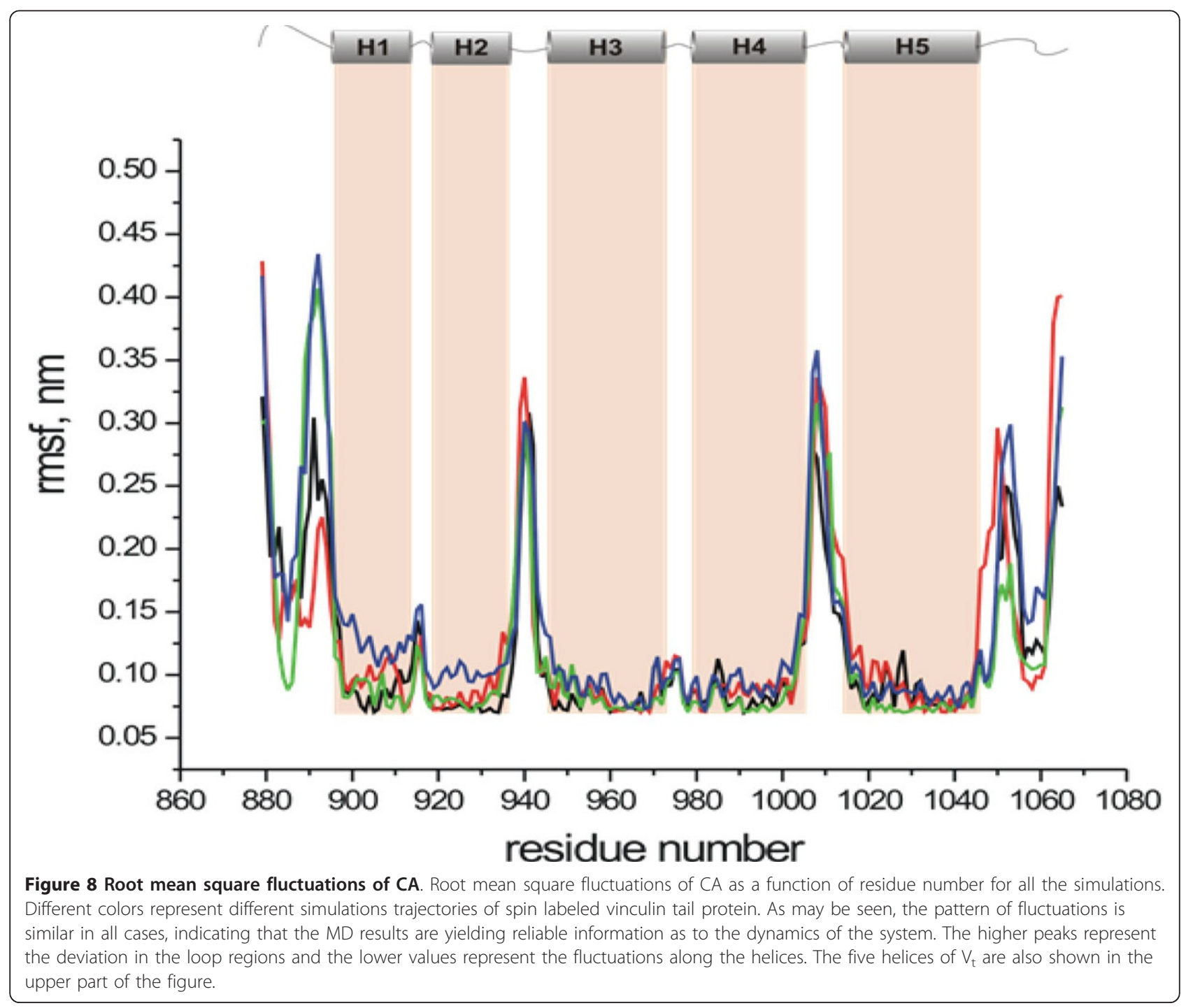




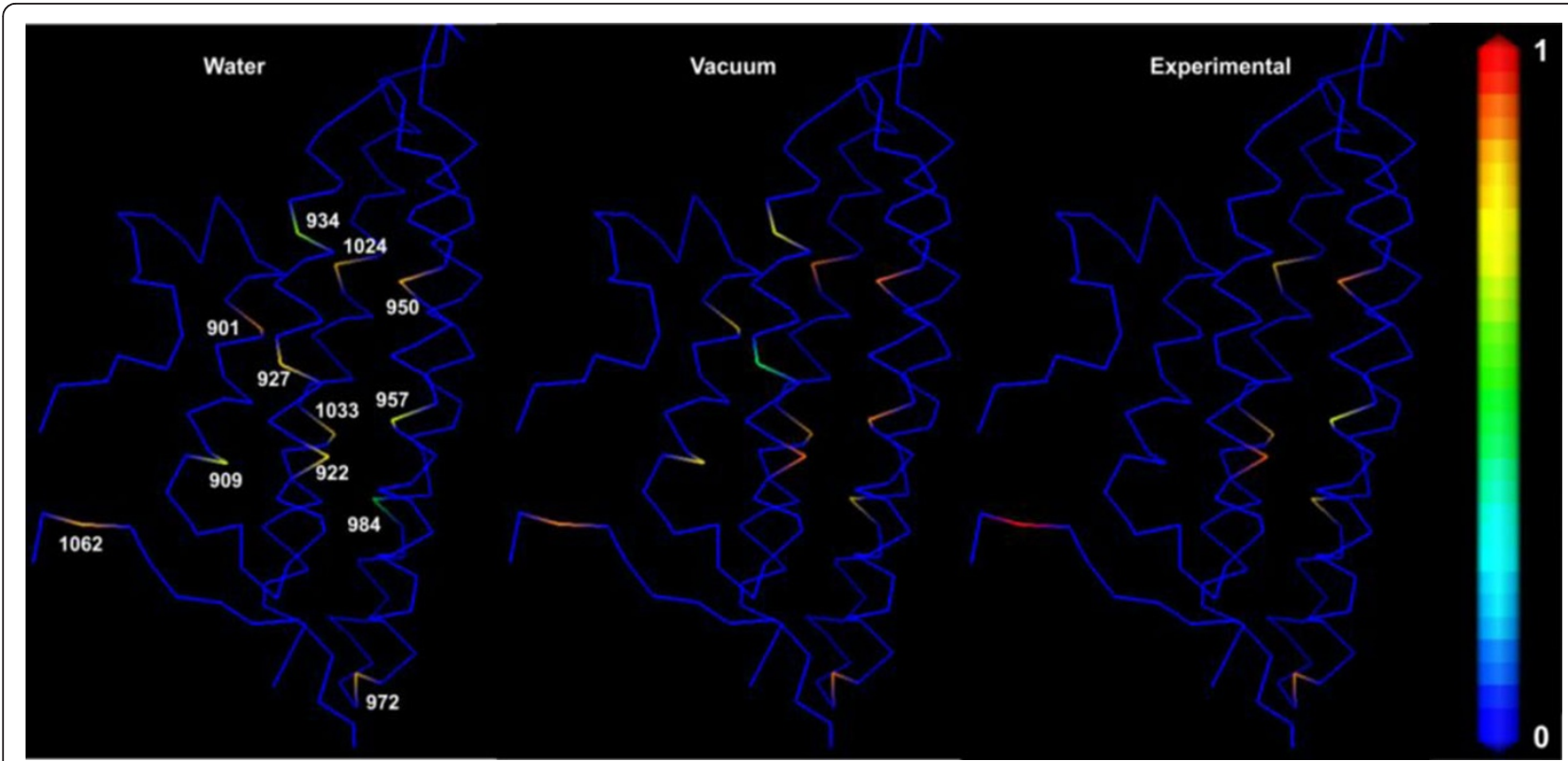

Figure 9 B factor based color scheme. Structure of vinculin tail with CA atoms represented in stick model colored by B-factor values. All the B-factor values in the coordinate file are set to zero and the mobility parameter values are replaced for the selected CA positions. Inverse line width values are used in the case of experimental values (multiplied by 1.5 times to facilitate comparison). Color coding bar is shown on the right side with the scale from zero (rigid) to one (mobile). However, experimental values for the positions 901, 909, 927 and 934 were not shown due to non availability.

of RMSF analysis and simulated EPR spectra. The results were comparable to EPR experiments. A correlation in the dynamics of the spin label mobility was found when mobility parameter values from MD simulations were compared with the EPR inverse line width data for the most of the spin-labeled sites of vinculin tail. To estimate the magnitude of helix displacements in the vinculin tail domain, distance distributions between pairs of spin label side chains were calculated and compared with experimental data. The MD simulations results in combination with EPR data show that the information contained in the spin label mobility provide a powerful means of mapping protein folds and their changes.

\section{Authors' contributions}

MNV participated in design of the study, carried out the molecular dynamics simulations, data analysis, comparison of MD data with EPR data and drafted the manuscript. KPV and FDI prepared mutants and carried out the EPR experiments. ANR participated in the statistical analysis. HJS conceived of the study, participated in its design, coordination and helped to draft the manuscript. All authors read and approved the final manuscript.

\section{Competing interests}

The authors declare that they have no competing interests.

\section{Acknowledgements}

Department of Science and Technology is highly appreciated for the funding through DST Ramanujan Fellowship award No. SR/S2/RJN-22/2011. We are very much thankful to Wolfgang Ziegler and Christoph Abe for all the valuable suggestions and extended help. We sincerely acknowledge everyone who directly or indirectly helped us to carryout this work.

\section{Declarations}

The publication costs for this article were funded by the corresponding author's sponsor institution, DST through Ramanujan fellowship award No. SR/S2/RJN-22/2011.

This article has been published as part of BMC Genomics Volume 14 Supplement 2, 2013: Selected articles from ISCB-Asia 2012. The full contents of the supplement are available online at http://www.biomedcentral.com/ bmcgenomics/supplements/14/S2.

\section{Author details}

${ }^{1}$ CABin division, DST Ramanujan Fellow, Indian Agricultural Statistics Research Institute, PUSA campus, New Delhi-110012, India. ${ }^{2}$ Department of Physics, University of Osnabrueck, Barbara strasse-7, D49076-Osnabrueck, Germany. ${ }^{3}$ IZKF Leipzig, Faculty of Medicine, University of Leipzig, Liebigstr. 21, D-04103 Leipzig, Germany.

\section{Published: 15 February 2013}

\section{References}

1. Ziegler $W H$, Liddington RC, Critchley DR: The structure and regulation of vinculin. Trends Cell Biol 2006, 16:453-460.

2. DeMali KA: Vinculin - a dynamic regulator of cell adhesion. TRENDS in Biochem Sci 2004, 29:11.

3. Ziegler $\mathrm{WH}$ : The cytoskeletal connection: understanding adaptor proteins. In Cell Migration: Signalling and Mechanisms. Transl Res Biomed. Volume 2. Basel, Karger;Entschladen F, Zänker KS 2010:136-162.

4. Jockusch BM, Riidiger M: Crosstalk between cell adhesion molecules: vinculin as a paradigm for regulation by conformation. Trends Cell Biol $1996,6: 311-315$

5. Izard T, et al: Vinculin activation by talin through helical bundle conversion. Nature 2004, 427:171-175.

6. Bakolitsa $C$, et al: Structural basis for vinculin activation at sites of cell adhesion. Nature 2004, 430:583-6. 
7. Bakolitsa C, de Pereda JM, Bagshaw CR, Critchley DR, Liddington RC: Crystal structure of the vinculin tail suggests a pathway for activation. Cell 1999, 99:603-613.

8. Johnson RP, Niggli V, Durrer P, Craig SW: A conserved motif in the tail domain of vinculin mediates association with and insertion into acidic phospholipid bilayers. Biochem 1998, 37:10211-222.

9. Niggli V: Structural properties of lipid-binding sites in cytoskeletal proteins. Trends Biochem Science 2001, 26:604-11.

10. DeMali KA, Barlow CA, Burridge K: Recruitment of the Arp2/3 complex to vinculin: coupling membrane protrusion to matrix adhesion. J Cell Biol 2002, 159:881-91.

11. Miller GJ, Dunn SD, Ball EH: Interaction of the $\mathrm{N}$ - and C-terminal domains of vinculin: characterization and mapping studies. J Biol Chem 2001, 276:11729-11734.

12. McGregor A, Blanchard AD, Rowe AJ, Critchley DR: Identification of the vinculin-binding site in the cytoskeletal protein a-actinin. Biochem J 1994, 301:225-233

13. Johnson RP, Craig SW: An intramolecular association between the head and tail domains of vinculin modulates talin binding. J Biol Chem 1994, 269:12611-12619.

14. Johnson RP, Craig SW: F-actin binding site masked by the intramolecular association of vinculin head and tail domains. Nature 1995, 373:261-264.

15. Altenbach C, Marti T, Khorana HG, Hubbell WL: Transmembrane Protein Structure: Spin Labeling of Bacteriorhodopsin Mutants. Science 1990, 248:1088-1092

16. Altenbach C, Steinhoff H-J, Greenhalgh DA, Khorana HG, Hubbell WL: Factors That Determine the EPR-Spectra of Nitroxide Side-Chains in Spin-Labeled Proteins and Analysis by Molecular-Dynamics Simulation. Biophys 1994, 66.

17. Altenbach C, Yang K, Farrens DL, Farahbakhsh ZT, Khorana HG, Hubbell WL: Structural Features and Light-Dependent Changes in the Cytoplasmic Interhelical E-F Loop Region of Rhodopsin: A Site-Directed Spin-Labeling Study. Biochemistry 1996, 35:12470-12478.

18. Bordignon E, Steinhoff H-J: Membrane protein structure and dynamics studied by site-directed spin labeling ESR. In Biological Magnetic Resonance 27 - ESR Spectroscopy in Membrane Biophysics Hemminga MA Berliner LJ 2007, 129-164.

19. Pfeiffer M, Rink T, Gerwert K, Steinhoff H-J: Site-directed Spin-labeling Reveals the orientation of the Amino Acid Side-chains in the E-F Loop of Bacteriorhodopsin. J Mol Biol 1999, 287:163-171.

20. Abe Christoph, Franziska D, Prasad Gajula, Monique B, Vogel KP, Maurice Gl, Susanne I, Wolfgang HG, Steinhoff H-J: Monomeric and Dimeric Conformation of the Vinculin Tail Five-Helix Bundle in Solution Determined by EPR-Spectroscopy. Biophysical Journal 2011, 101(7):1772-1780

21. Gajula P, Borovykh IV, Beier C, Shkuropatova T, Gast P, Steinhoff H-J: Spinlabeled photosynthetic reaction centers from Rhodobacter sphaeroides studied by electron paramagnetic resonance spectroscopy and molecular dynamics simulations. Applied Magnetic Resonance 2007, 31:167-178.

22. Borovykh V, Gajula P, Huber M, Gast P, Stenhoff H-J: Distance between a native cofactor and a spin label in the reaction centre of Rhodobacter sphaeroides by a two-frequency pulsed electron paramagnetic resonance method and molecular dynamics simulations. Journal of magnetic resonance 2006, 180:178-185.

23. Klare JP, Steinhoff H-J: Spin Labeling EPR. Photosynth Res 2009, 102:377-90.

24. Steinhoff HJ, Hubbell WL: Calculation of electron paramagnetic resonance spectra from Brownian dynamics trajectories: application to nitroxide side chains in proteins. Biophys J 1996, 71(4):2201-12

25. LaConte EWLeslie, Voelz Vincent, Nelson Wendy, Enz Michael, Thomas DDavid: Molecular Dynamics Simulation of Site-Directed Spin Labeling: Experimental Validation in Muscle Fibers. Biophysical Journal 2002, 83:1854-1866.

26. Beier C, Steinhoff HJ: A structure-based simulation approach for electron paramagnetic resonance spectra using molecular and stochastic dynamics simulations. Biophys J 2006, 91(7):2647-64.

27. Prasad Gajula MNV: PhD Thesis. [http://repositorium.uni-osnabrueck.de/ bitstream/urn:nbn:de:gbv:700-2008041631/2/E-Diss781_thesis.pdf].

28. Palmer SM, Playford MP, Craig SW, Schaller MD, Campbell SL: Lipid binding to the tail domain of vinculin: specificity and the role of the $\mathrm{N}$ and $\mathrm{C}$ termini. J Biol Chem 2009, 284:7223-7231.
29. Lindahl E, Hess B, van der Spoel D: GROMACS 3.0: A package for molecular simulation and trajectory analysis. J Mol Mod 2001, 7:306-317.

30. van der Spoel D, Lindahl E, Hess B, Groenhof G, Mark AE, Berendsen HJC: GROMACS: Fast, Flexible and Free. J Comp Chem 2005, 26:1701-1718.

31. Shen K, Tolbert CE, Guilluy C, Swaminathan VS, Berginski ME, Burridge K, Superfine R, Campbell SL: The vinculin C-terminal hairpin mediates F-actin bundle formation, focal adhesion, and cell mechanical properties. J Biol Chem 2011, 286:45103-45115.

32. Critchley $D R$, et al: Cytoskeletal proteins talin and vinculin in integrinmediated adhesion. Biochem Soc Trans 2004, 32:831-836.

33. Saunders RM, Holt MR, Jennings L, Sutton DH, Barsukov IL, Bobkov $A$, Liddington RC, Adamson EA, Dunn GA, *Critchley DR: *Role of vinculin in regulating focal adhesion turnover. Eur J Cell Biol 2006, 85:487-500

doi:10.1186/1471-2164-14-S2-S4

Cite this article as: Prasad Gajula et al:: How far in-silico computing meets real experiments. A study on the structure and dynamics of spin labeled vinculin tail protein by molecular dynamics simulations and EPR spectroscopy. BMC Genomics 2013 14(Suppl 2):S4.

\section{Submit your next manuscript to BioMed Central and take full advantage of:}

- Convenient online submission

- Thorough peer review

- No space constraints or color figure charges

- Immediate publication on acceptance

- Inclusion in PubMed, CAS, Scopus and Google Scholar

- Research which is freely available for redistribution

C BioMed Central 